\title{
Sharp one-weight and two-weight bounds for maximal operators
}

by

Kabe Moen (Lawrence, KS)

\begin{abstract}
We investigate the boundedness of the fractional maximal operator with respect to a general basis on weighted Lebesgue spaces. We characterize the boundedness of these operators for one-weight and two-weight inequalities extending the work of Jawerth. A new two-weight testing condition for the fractional maximal operator on a general basis is introduced extending the work of Sawyer for the basis of cubes. We also find the sharp dependence in the two-weight case between the operator norm and the testing condition of Sawyer. Finally, our approach leads to a new proof of Buckley's sharp estimate for the Hardy-Littlewood maximal function.
\end{abstract}

1. Introduction. Consider the family of maximal operators defined by

$$
M_{\alpha}^{\mathcal{B}} f(x)=\sup _{B \ni x} \frac{1}{|B|^{1-\alpha / n}} \int_{B}|f(y)| d y, \quad 0 \leq \alpha<n,
$$

where the supremum is taken over all $B$ containing $x$ and belonging to some basis of open sets, $\mathcal{B}$. When $\mathcal{B}=\mathcal{Q}$, the basis of cubes in $\mathbb{R}^{n}$, we drop the superscript and simply write $M_{\alpha}$. In this case we have the familiar operators, $M$, the Hardy-Littlewood maximal operator $(\alpha=0)$ and fractional maximal operator, $M_{\alpha}(0<\alpha<n)$.

The weighted inequalities for these operators are of the form

$$
\left(\int_{\mathbb{R}^{n}}\left(M_{\alpha}^{\mathcal{B}} f(x) w(x)\right)^{q} d x\right)^{1 / q} \leq C\left(\int_{\mathbb{R}^{n}}(|f(x)| w(x))^{p} d x\right)^{1 / p}
$$

for a single weight $w$, and

$$
\left(\int_{\mathbb{R}^{n}} M_{\alpha}^{\mathcal{B}} f(x)^{q} u(x) d x\right)^{1 / q} \leq C\left(\int_{\mathbb{R}^{n}}|f(x)|^{p} v(x) d x\right)^{1 / p}
$$

for a pair of weights $(u, v)$. In this paper we examine these inequalities along with the dependence of the operator norm of $M_{\alpha}^{\mathcal{B}}$ on the weights.

2000 Mathematics Subject Classification: Primary 42B25.

Key words and phrases: maximal functions, weights. 
For the basis of cubes much is known about the one-weight inequalities. The seminal work of Muckenhoupt [10] introduced the $A_{p}$ classes of weights and characterized boundedness of the Hardy-Littlewood maximal operator on $L^{p}(w)$. Namely, he showed that for $1<p<\infty$,

$$
\|M f\|_{L^{p}(w)} \leq C\|f\|_{L^{p}(w)}
$$

if and only if $w$ belongs to the class $A_{p}$, i.e.,

$$
[w]_{A_{p}}=\sup _{Q}\left(\frac{1}{|Q|} \int_{Q} w(x) d x\right)\left(\frac{1}{|Q|} \int_{Q} w(x)^{1-p^{\prime}} d x\right)^{p-1}<\infty .
$$

A nice review of the history of one-weight inequalities for the Hardy-Littlewood maximal function can be found in Jawerth [7] or Lerner [9].

When $0 \leq \alpha<n$, Muckenhoupt-Wheeden [11] characterized the boundedness of $M_{\alpha}$ in terms of a similar condition. They showed that for $1<p<$ $n / \alpha$ and $q$ defined by $1 / q=1 / p-\alpha / n$,

$$
\left\|M_{\alpha} f w\right\|_{L^{q}} \leq C\|f w\|_{L^{p}}
$$

if and only if $w \in A_{p, q}$, i.e.,

$$
[w]_{A_{p, q}}=\sup _{Q}\left(\frac{1}{|Q|} \int_{Q} w(x)^{q} d x\right)\left(\frac{1}{|Q|} \int_{Q} w(x)^{-p^{\prime}} d x\right)^{q / p^{\prime}}<\infty .
$$

Notice that $[w]_{A_{p, q}}=\left[w^{q}\right]_{A_{1+q / p^{\prime}}}$, so $w \in A_{p, q}$ if and only $w^{q} \in A_{1+q / p^{\prime}}$.

For the case $\alpha=0$, Jawerth [7] extended this theory to general bases. He proved the following theorem (see Section 2 below for pertinent definitions).

Theorem A. Let $\mathcal{B}$ be a basis, w a weight, and $1<r<\infty$. Then

$$
\left\{\begin{array}{l}
M^{\mathcal{B}}: L^{r}(w) \rightarrow L^{r}(w) \\
M^{\mathcal{B}}: L^{r^{\prime}}(\sigma) \rightarrow L^{r^{\prime}}(\sigma)
\end{array}\right.
$$

if and only $w$ satisfies the $A_{p}$ condition with respect to $\mathcal{B}$, and

$$
\left\{\begin{array}{l}
M_{w}^{\mathcal{B}}: L^{r^{\prime}}(w) \rightarrow L^{r^{\prime}}(w), \\
M_{\sigma}^{\mathcal{B}}: L^{r}(\sigma) \rightarrow L^{r}(\sigma)
\end{array}\right.
$$

Lerner [9] gave a simple approach to the one-weight theory which yields proofs of Muckenhoupt's and Jawerth's theorems and gives sharp constants. The case $\alpha \neq 0$ for a general basis apparently has not been considered in the literature before.

When $\mathcal{B}=\mathcal{Q}$ the two-weight theory is again well known. Sawyer [12] classified the two-weight boundedness of $M_{\alpha}$ in terms of a "testing condition". He proved that $\left\|M_{\alpha} f\right\|_{L^{q}(u)} \leq C\|f\|_{L^{p}(v)}$ if and only if the pair of 
weights $(u, v)$ satisfies

$$
\left(\int_{Q} M_{\alpha}\left(\chi_{Q} v^{1-p^{\prime}}\right)(x)^{q} u(x) d x\right)^{1 / q} \leq C\left(\int_{Q} v(x)^{1-p^{\prime}} d x\right)^{1 / p} .
$$

When $\alpha=0$ and $p=q$ the following version of Sawyer's theorem for $M^{\mathcal{B}}$ is also due to Jawerth [7].

TheOREM B. Let $1<p<\infty$ and $(u, v)$ be a couple of weights, $\sigma=v^{1-p^{\prime}}$, and assume that $M_{\sigma}^{\mathcal{B}}$ is bounded on $L^{p}(\sigma)$. Then

$$
M^{\mathcal{B}}: L^{p}(v) \rightarrow L^{p}(u)
$$

if and only if $M^{\mathcal{B}}$ satisfies the testing condition

$$
\int_{G} M^{\mathcal{B}}\left(\chi_{G} \sigma\right)(x)^{p} u(x) d x \leq C \sigma(G)
$$

for all $G$ that are unions of sets in $\mathcal{B}$.

Recently, much attention has been paid to obtaining sharp bounds for the operator norm of various operators on weighted spaces. We briefly summarize the history of this problem for the Hardy-Littlwood maximal operator.

Buckley [1] obtained the sharp bound on the operator norm of the Hardy-Littlewood maximal function in terms of the $A_{p}$ constant, showing that

$$
\|M\| \leq C[w]_{A_{p}^{1 /(p-1)}}^{1}
$$

The sharpness of (1.1) follows from computing the constants for appropriate families of power functions and power weights. Buckley's paper mentions that careful examination of the proof by Coifman-Fefferman [3] also yields the bound (1.1). However, (1.1) can also be obtained in number of other manners. These include: the short proof given by Christ-Fefferman [2] and the very short (six lines) proof by Lerner [9]. Moreover, we show in this article that (1.1) can also be obtained by combining the two-weight result of Sawyer [12] with the arguments of Hunt-Kurtz-Neugebauer [6].

For $\alpha>0$ the sharp bound on the operator norm of $M_{\alpha}$ was recently found. In [8] the author, Pérez, and Torres obtained the analogous result for $M_{\alpha}: L^{p}\left(w^{p}\right) \rightarrow L^{q}\left(w^{q}\right)$, showing the sharp constant is

$$
\left\|M_{\alpha}\right\|_{L^{p}\left(w^{p}\right) \rightarrow L^{q}\left(w^{q}\right)} \leq C[w]_{A_{p, q}}^{\frac{p^{\prime}}{q}(1-\alpha / n)} .
$$

The sharp bound for two-weight inequalities is not known, but in this paper we introduce a new testing condition for $M_{\alpha}$ that is sufficient for the two-weight inequality. When $\alpha=0$ and $p=q$ the new testing condition (4.7) is the same as Sawyer's condition. An advantage of our approach is that it yields the sharp bound on the two-weight operator norm of the 
Hardy-Littlewood maximal operator. In Theorem 5.1 we examine the relationship between the $A_{p, q}$ constant and the constant in the testing condition. This leads to a new proof of (1.2) (for a detailed discussion see the end of Section 5). Also, we examine previously unknown aspects of the theory related to the family of operators $M_{\alpha}^{\mathcal{B}}$. Our main results are analogous to Theorems A and B.

The layout of the paper will be as follows. In Section 2 we present some technical definitions and lemmata. In Section 3 we present our main result concerning the one-weight theory. Section 4 contains the two-weight theory including an extension of Theorem B and the new testing condition which yields the sharp constants in the one-weight case. Finally, Section 5 contains the relationship between the new testing condition and the $A_{p}$ conditions for one weight and some remarks about sharp constants. As a consequence of our methods, when $\alpha=0$ we find the sharp two-weight bound and we uncover a new proof of inequality (1.1).

2. Preliminaries. Given an exponent $1 \leq p \leq \infty, p^{\prime}$ will denote the dual exponent of $p$ defined by the equation $1 / p+1 / p^{\prime}=1$ with the usual modifications for the end points. For non-negative $w, L^{p}(w)$ will denote the Lebesgue space normed by

$$
\|f\|_{L^{p}(w)}=\left(\int_{\mathbb{R}^{n}}|f|^{p} w d x\right)^{1 / p} .
$$

Given two Banach spaces $X$ and $Y$ we will use the notation $T: X \rightarrow Y$ to mean that $T$ is a bounded operator from $X$ to $Y$ i.e.

$$
\|T x\|_{Y} \leq C\|x\|_{X}
$$

for all $x \in X$. The smallest constant $C$ which satisfies (2.1) will denote the operator norm of $T,\|T\|_{X \rightarrow Y}$. When it is clear we will just write $\|T\|$.

A basis, denoted $\mathcal{B}$, is a collection of open sets in $\mathbb{R}^{n}$. Two important bases of interest are $\mathcal{Q}$, the basis of cubes in $\mathbb{R}^{n}$, and $\mathcal{D}$, the basis of dyadic cubes in $\mathbb{R}^{n}$. Dyadic cubes are cubes of the form $2^{k}\left(m+[0,1)^{n}\right)$ where $k \in \mathbb{Z}$ and $m \in \mathbb{Z}^{n}$. A weight (with respect to a given basis) is a non-negative function $w$ satisfying $w(B)<\infty$ for all $B \in \mathcal{B}$. We define the weighted fractional maximal operator with respect to the basis $\mathcal{B}$ by

$$
M_{\alpha, w}^{\mathcal{B}} f(x)=\sup _{B \ni x} \frac{1}{w(B)^{1-\alpha / n}} \int_{B}|f(y)| w(y) d y, \quad 0 \leq \alpha<n,
$$

where the supremum is taken over all $B \in \mathcal{B}$ for which $x \in B$ and $w(B)>0$, and is defined to be zero if $w(B)=0$ for all $B \in \mathcal{B}$ that contain $x$. When $w \equiv 1$ we drop the subscript $w$ and write $M_{\alpha}^{\mathcal{B}}$. The class $A_{p}^{\mathcal{B}}$ is composed of 
the weights $w$ that satisfy

$$
[w]_{A_{p}^{\mathcal{B}}}=\sup _{B \in \mathcal{B}}\left(\frac{1}{|B|} \int_{B} w(x) d x\right)\left(\frac{1}{|B|} \int_{B} w(x)^{1-p^{\prime}} d x\right)^{p-1}<\infty .
$$

Similarly, for $1<p \leq q<\infty$ we define $A_{p, q}^{\mathcal{B}}$ to be the class of weights $w$ that satisfy

$$
[w]_{A_{p, q}^{\mathcal{B}}}=\sup _{B \in \mathcal{B}}\left(\frac{1}{|B|} \int_{B} w(x)^{q} d x\right)\left(\frac{1}{|B|} \int_{B} w(x)^{-p^{\prime}} d x\right)^{q / p^{\prime}}<\infty .
$$

We start with an elementary lemma which will be useful in the one-weight and two-weight theorems.

LEMMA 2.1. Let $0 \leq \alpha<n$, and $v$ be a weight. Then the operator $M_{\alpha, v}^{\mathcal{D}}$ satisfies

$$
v\left(\left\{x:\left|M_{\alpha, v}^{\mathcal{D}} f(x)\right|>\lambda\right\}\right)^{1-\alpha / n} \leq \frac{\|f\|_{L^{1}(v)}}{\lambda}
$$

and

$$
\left\|M_{\alpha, v}^{\mathcal{D}} f\right\|_{L^{q}(v)} \leq C_{n, p}\|f\|_{L^{p}(v)}
$$

for all $1<p \leq n / \alpha$ and $q$ defined by $1 / q=1 / p-\alpha / n$. Furthermore, the constant $C_{n, p}$ is independent of $v$.

Proof. The proof is by interpolation. By Hölder's inequality with exponents $n / \alpha$ and $(n / \alpha)^{\prime}=n /(n-\alpha)$,

$$
\begin{aligned}
\frac{1}{v(Q)^{1-\alpha / n}} \int_{Q}|f| v d x & \leq \frac{1}{v(Q)^{1-\alpha / n}}\left(\int_{Q}|f|^{n / \alpha} v d x\right)^{\alpha / n}\left(\int_{Q} v d x\right)^{1-\alpha / n} \\
& \leq\|f\|_{L^{n / \alpha}(v)} .
\end{aligned}
$$

It follows that

$$
\left\|M_{\alpha, v}^{\mathcal{D}} f\right\|_{L^{\infty}(v)} \leq\|f\|_{L^{n / \alpha}(v)}
$$

For the weak- $(1, n /(n-\alpha))$ estimate, using the properties of dyadic cubes we may write

$$
\left\{x: M_{\alpha, v}^{\mathcal{D}} f(x)>\alpha\right\}=\bigcup_{j} Q_{j},
$$

where $Q_{j}$ are disjoint dyadic cubes that satisfy

$$
\frac{1}{v\left(Q_{j}\right)^{1-\alpha / n}} \int_{Q_{j}}|f| v d x>\lambda
$$


Hence,

$$
\begin{aligned}
v\left(\left\{M_{\alpha, v}^{\mathcal{D}} f>\lambda\right\}\right) & =\sum_{j} v\left(Q_{j}\right) \leq \sum_{j}\left(\frac{1}{\lambda} \int_{Q_{j}}|f| v d x\right)^{n /(n-\alpha)} \\
& \leq\left(\sum_{j} \frac{1}{\lambda} \int_{Q_{j}}|f| v d x\right)^{n /(n-\alpha)} \leq\left(\frac{1}{\lambda}\|f\|_{L^{1}(v)}\right)^{n /(n-\alpha)} .
\end{aligned}
$$

Thus, by interpolation,

$$
\left\|M_{\alpha, v}^{\mathcal{D}} f\right\|_{L^{q}(v)} \leq C_{n, p}\|f\|_{L^{p}(v)},
$$

where $1 / q=1 / p-\alpha / n$.

Finally, we state one more lemma that allows us to transfer results from the the basis $\mathcal{D}$ to the basis $\mathcal{Q}$. We state the lemma without proof as the case $\alpha=0$ can be found in the book by García-Cuerva and Rubio de Francia [5, p. 431]. It is based on the ideas of Fefferman and Stein [4], and the proof for general $\alpha$ is a straightforward generalization.

Lemma 2.2. Let $0<q<\infty, u$ be a non-negative function, and $\tau_{t}$ be the shift operator $\tau_{t} g(x)=g(x-t)$. Then

$$
\left\|M_{\alpha} f\right\|_{L^{q}(u)} \leq C_{n} \sup _{t}\left\|\tau_{-t} \circ M_{\alpha}^{\mathcal{D}} \circ \tau_{t} f\right\|_{L^{q}(u)},
$$

where $C_{n}$ depends only on the dimension.

3. One-weight results. We now state our main one-weight result for the fractional maximal operator with respect to a general basis. The following theorem is analogous to Theorem A.

Theorem 3.1. Suppose $0 \leq \alpha<n, 1<p<n / \alpha, q$ is the number defined by $1 / q=1 / p-\alpha / n$, and $w$ is a weight. Let $u=w^{q}, \sigma=w^{-p^{\prime}}$ and $r=1+q / p^{\prime}$. Then

$$
\begin{aligned}
& M_{\alpha}^{\mathcal{B}}: L^{p}\left(w^{p}\right) \rightarrow L^{q}\left(w^{q}\right), \\
& M_{\alpha}^{\mathcal{B}}: L^{q^{\prime}}\left(w^{-q^{\prime}}\right) \rightarrow L^{p^{\prime}}\left(w^{-p^{\prime}}\right), \\
& M^{\mathcal{B}}: L^{r}(u) \rightarrow L^{r}(u), \\
& M^{\mathcal{B}}: L^{r^{\prime}}(\sigma) \rightarrow L^{r^{\prime}}(\sigma),
\end{aligned}
$$

if and only if $w \in A_{p, q}^{\mathcal{B}}$, and

$$
\begin{aligned}
M_{\alpha, \sigma}^{\mathcal{B}} & : L^{p}(\sigma) \rightarrow L^{q}(\sigma), \\
M_{\alpha, u}^{\mathcal{B}} & : L^{q^{\prime}}(u) \rightarrow L^{p^{\prime}}(u), \\
M_{\sigma}^{\mathcal{B}} & : L^{r}(\sigma) \rightarrow L^{r}(\sigma), \\
M_{u}^{\mathcal{B}}: & L^{r^{\prime}}(u) \rightarrow L^{r^{\prime}}(u) .
\end{aligned}
$$


Furthermore, we have the following operator norm inequalities:

$$
\left\|M_{\alpha}^{\mathcal{B}}\right\|_{L^{p}\left(w^{p}\right) \rightarrow L^{q}\left(w^{q}\right)} \leq[w]_{A_{p, q}^{\mathcal{B}}}^{\frac{p^{\prime}}{q}(1-\alpha / n)}\left\|M_{u}^{\mathcal{B}}\right\|_{L^{r^{\prime}}(u) \rightarrow L^{r^{\prime}}(u)}^{\frac{p^{\prime}}{q}(1-\alpha / n)}\left\|M_{\alpha, \sigma}^{\mathcal{B}}\right\|_{L^{p}(\sigma) \rightarrow L^{q}(\sigma)}
$$

and

$$
\begin{aligned}
\left\|M_{\alpha}^{\mathcal{B}}\right\|_{L^{q^{\prime}}\left(w^{-q^{\prime}}\right)} \rightarrow L^{p^{\prime}\left(w^{-p^{\prime}}\right)} & \\
& \leq\left[w^{-1}\right]_{A_{q^{\prime}, p^{\prime}}^{p^{\prime}}(1-\alpha / n)}^{\frac{p^{\prime}}{p^{\prime}}}\left\|M_{\sigma}^{\mathcal{B}}\right\|_{L^{r}(\sigma) \rightarrow L^{r}(\sigma)}^{\frac{q}{p^{\prime}}(1-\alpha / n)}\left\|M_{\alpha, u}^{\mathcal{B}}\right\|_{L^{q^{\prime}}(u) \rightarrow L^{p^{\prime}}(u)} .
\end{aligned}
$$

REMARK 3.2. Note that when $\alpha=0$, and hence $q=p$, many of the conditions in Theorem 3.1 collapse. In such a case we have the following equivalent conditions: $(3.1)=(3.3),(3.2)=(3.4),(3.5)=(3.7)$, and $(3.6)=(3.8)$. However, this is just the renormalized $\left(w \mapsto w^{p}\right)$ version of Theorem A and hence we exclude this case from the proof of Theorem 3.1.

REMARK 3.3. Since

$$
w \in A_{p, q}^{\mathcal{B}} \Leftrightarrow w^{q} \in A_{1+q / p^{\prime}}^{\mathcal{B}},
$$

if we apply Theorem A with exponent $r=1+q / p^{\prime}$ (notice $1<r<\infty$ ) we have the equivalence

$$
(3.3),(3.4) \Leftrightarrow(3.8),(3.7) \text {, and } w \in A_{p, q}^{\mathcal{B}} .
$$

Here are some guidelines for the conditions in Theorem 3.1. We will show that

$$
\text { (3.5), (3.8), and } w \in A_{p, q}^{\mathcal{B}} \Rightarrow(3.1)
$$

and

$$
\text { (3.6), (3.7), and } w \in A_{p, q}^{\mathcal{B}} \Rightarrow(3.2) .
$$

For the reverse implications, any of conditions (3.1) to (3.4) implies that $w \in A_{p, q}^{\mathcal{B}}$ and for the maximal functions we will show

$$
(3.1),(3.4) \Rightarrow(3.5) \text { and }(3.2),(3.8) \Rightarrow(3.6) \text {. }
$$

Proof of Theorem 3.1. Suppose $\alpha>0$. We only prove that (3.5), (3.8), and $w \in A_{p, q}^{\mathcal{B}}$ implies (3.1); also, (3.1) and (3.4) implies (3.5), as the other implications stated in Remark 3.3 are similar. We follow some ideas in [9]. Suppose that $M_{\alpha, \sigma}^{\mathcal{B}}$ and $M_{u}^{\mathcal{B}}$ are as in (3.5) and (3.8) with operator norms $\left\|M_{\alpha, \sigma}^{\mathcal{B}}\right\|_{p, q},\left\|M_{u}^{\mathcal{B}}\right\|_{r^{\prime}}$, and $w \in A_{p, q}^{\mathcal{B}}$. Notice we may write the $A_{p, q}^{\mathcal{B}}$ constant as

$$
[w]_{A_{p, q}^{\mathcal{B}}}=\sup _{B} \frac{u(B)}{|B|}\left(\frac{\sigma(B)}{|B|}\right)^{q / p^{\prime}} .
$$


Let $x \in \mathbb{R}^{n}$ and $B \in \mathcal{B}$ be a set containing $x$. Let $r=1+q / p^{\prime}$ so that $r^{\prime}=1+p^{\prime} / q$. Then, using the equation $1-\alpha / n=1 / q+1 / p^{\prime}$, we have

$$
\begin{aligned}
& \frac{1}{|B|^{1-\alpha / n}} \int_{B}|f| d x=\frac{u(B)^{\frac{p^{\prime}}{q}(1-\alpha / n)} \sigma(B)^{1-\alpha / n}}{|B|^{\left(1+p^{\prime} / q\right)(1-\alpha / n)}} \\
& \times \frac{|B|^{\left(1+p^{\prime} / q\right)(1-\alpha / n)}}{u(B)^{\frac{p^{\prime}}{q}(1-\alpha / n)} \sigma(B)^{1-\alpha / n}} \frac{1}{|B|^{1-\alpha / n}} \int_{B}|f| d x \\
& \leq[w]_{A_{p, q}^{\mathcal{B}}}^{\frac{p^{\prime}}{q}(1-\alpha / n)}\left(\frac{|B|}{u(B)}\left(\frac{1}{\sigma(B)^{1-\alpha / n}} \int_{B}|f| \sigma^{-1} \sigma d x\right)^{q / r^{\prime}}\right)^{r^{\prime} / q} \\
& \leq[w]_{A_{p, q}^{\mathcal{B}}}^{\frac{p^{\prime}}{q}(1-\alpha / n)}\left(\frac{1}{u(B)} \int_{B}\left(M_{\alpha, \sigma}^{\mathcal{B}}\left(f \sigma^{-1}\right)(x)^{q / r^{\prime}} u^{-1} u d x\right)^{r^{\prime} / q} .\right.
\end{aligned}
$$

Taking the supremum we have the pointwise estimate

$$
M_{\alpha}^{\mathcal{B}} f(x) \leq[w]_{A_{p, q}^{\mathcal{p}}}^{\frac{p^{\prime}}{q}(1-\alpha / n)}\left(M_{u}^{\mathcal{B}}\left(M_{\alpha, \sigma}^{\mathcal{B}}\left(f \sigma^{-1}\right)^{q / r^{\prime}} u^{-1}\right)(x)\right)^{r^{\prime} / q} .
$$

Hence,

$$
\begin{aligned}
\left\|M_{\alpha}^{\mathcal{B}} f w\right\|_{L^{q}} & \leq[w]_{A_{p}^{\mathcal{B}},}^{\frac{p^{\prime}}{q}(1-\alpha / n)}\left(\int_{\mathbb{R}^{n}}\left(M_{u}^{\mathcal{B}}\left(M_{\alpha, \sigma}^{\mathcal{B}}\left(f \sigma^{-1}\right)^{q / r^{\prime}} u^{-1}\right)(x)\right)^{r^{\prime}} u d x\right)^{1 / q} \\
& \leq[w]_{A_{p, q}^{\mathcal{B}}}^{\frac{p^{\prime}}{q}(1-\alpha / n)}\left\|M_{u}^{\mathcal{B}}\right\|_{r^{\prime}}^{r^{\prime} / q}\left(\int_{\mathbb{R}^{n}}\left(M_{\alpha, \sigma}^{\mathcal{B}}\left(f \sigma^{-1}\right)(x)\right)^{q} u^{-r^{\prime}} u d x\right)^{1 / q} \\
& =[w]_{A_{p, q}^{\mathcal{B}}}^{\frac{p^{\prime}}{q}(1-\alpha / n)}\left\|M_{u}^{\mathcal{B}}\right\|_{r^{\prime}}^{r^{\prime} / q}\left(\int_{\mathbb{R}^{n}}\left(M_{\alpha, \sigma}^{\mathcal{B}}\left(f \sigma^{-1}\right)(x)\right)^{q} \sigma d x\right)^{1 / q} \\
& \leq[w]_{A_{p, q}^{\mathcal{B}}}^{\frac{p^{\prime}}{q}(1-\alpha / n)}\left\|M_{u}^{\mathcal{B}}\right\|_{r^{\prime}}^{\frac{p^{\prime}}{q}(1-\alpha / n)}\left\|M_{\alpha, \sigma}^{\mathcal{B}}\right\|_{p, q}\left(\int_{\mathbb{R}}|f|^{p} \sigma^{-p} \sigma d x\right)^{1 / p} \\
& =[w]_{A_{p, q}^{\mathcal{B}}}^{\frac{p^{\prime}}{q}(1-\alpha / n)}\left\|M_{u}^{\mathcal{B}}\right\|_{r^{\prime}}^{\frac{p^{\prime}}{q}(1-\alpha / n)}\left\|M_{\alpha, \sigma}^{\mathcal{B}}\right\|_{p, q}\|f w\|_{L^{p}},
\end{aligned}
$$

and we obtain (3.1) with the right bound.

Suppose now $M_{\alpha}^{B}$ and $M^{\mathcal{B}}$ are bounded as in (3.1) and (3.3) respectively. Notice that for any $B \in \mathcal{B}$, by Hölder's inequality, we have

$$
1=\frac{1}{|B|} \int_{B} w^{q / r} w^{-q / r} d x \leq\left(\frac{u(B)}{|B|}\right)^{1 / r}\left(\frac{\sigma(B)}{|B|}\right)^{1 / r^{\prime}},
$$

so

$$
\left(\frac{|B|}{\sigma(B)}\right)^{r} \leq\left(\frac{u(B)}{|B|}\right)^{r^{\prime}}
$$


With similar computations we have

$$
\begin{aligned}
\left(\frac{1}{\sigma(B)^{1-\alpha / n}} \int_{B} f \sigma d x\right)^{q} & =\left(\frac{|B|^{1-\alpha / n}}{\sigma(B)^{1-\alpha / n}}\right)^{q}\left(\frac{1}{|B|^{1-\alpha / n}} \int_{B} f \sigma d x\right)^{q} \\
& =\left(\frac{|B|}{\sigma(B)}\right)^{r}\left(\frac{1}{|B|^{1-\alpha / n}} \int_{B} f \sigma d x\right)^{q} \\
& \leq\left(\frac{u(B)}{|B|}\right)^{r^{\prime}}\left(\frac{1}{|B|^{1-\alpha / n}} \int_{B} f \sigma d x\right)^{q} \\
& =\left(\frac{u(B)}{|B|}\left(\frac{1}{|B|^{1-\alpha / n}} \int_{B} f \sigma d x\right)^{q / r^{\prime}}\right)^{r^{\prime}} \\
& \leq\left(\frac{1}{|B|} \int_{B} M_{\alpha}^{\mathcal{B}}(f \sigma)^{q / r^{\prime}} u d x\right)^{r^{\prime}} .
\end{aligned}
$$

Taking the supremum over all $B \in \mathcal{B}$ with $x \in B$ we have

$$
M_{\alpha, \sigma}^{\mathcal{B}} f(x)^{q} \leq M^{\mathcal{B}}\left(M_{\alpha}^{\mathcal{B}}(f \sigma)^{q / r^{\prime}} u\right)(x)^{r^{\prime}} .
$$

Hence,

$$
\begin{aligned}
\left\|M_{\alpha, \sigma}^{\mathcal{B}} f\right\|_{L^{q}(\sigma)} & \leq\left\|M^{\mathcal{B}}\left(M_{\alpha}^{\mathcal{B}}(f \sigma)^{q / r^{\prime}} u\right)\right\|_{L^{r^{\prime}}(\sigma)}^{r^{\prime} / q} \\
& \leq C\left\|M_{\alpha}^{\mathcal{B}}(f \sigma)\right\|_{L^{q}(u)} \leq C\|f \sigma\|_{L^{p}\left(w^{p}\right)}=C\|f\|_{L^{p}(\sigma)} .
\end{aligned}
$$

When $\mathcal{B}=\mathcal{D}, M_{\sigma}^{\mathcal{D}}$ and $M_{u}^{\mathcal{D}}$ are automatically bounded, with operator norms independent of $w$ and in light of Lemma 2.1 so are $M_{\alpha, \sigma}^{\mathcal{D}}$ and $M_{\alpha, u}^{\mathcal{D}}$. Hence we have as a corollary the following dyadic version of the result found in $[11]$.

Corollary 3.4. Suppose $1<p<n / \alpha$, and $q$ is defined by $1 / q=$ $1 / p-\alpha / n$. Then $M_{\alpha}^{\mathcal{D}}: L^{p}\left(w^{p}\right) \rightarrow L^{q}\left(w^{q}\right)$ if and only if $w \in A_{p, q}^{\mathcal{D}}$ with

$$
\left\|M_{\alpha}^{\mathcal{D}}\right\| \leq C[w]_{A_{p, q}^{\mathcal{D}}}^{\frac{p^{\prime}}{q}(1-\alpha / n)}
$$

4. Two-weight theory. For the two-weight theory we are looking for conditions on pairs of weights $(u, v)$ so that

$$
\left(\int_{\mathbb{R}^{n}} M_{\alpha}^{\mathcal{B}} f(x)^{q} u(x) d x\right)^{1 / q} \leq C\left(\int_{\mathbb{R}^{n}}|f(x)|^{p} v(x) d x\right)^{1 / p} .
$$

We have the following theorem analogous to Theorem B.

Theorem 4.1. Let $\mathcal{B}$ be a basis, $0 \leq \alpha<n, 1<p \leq q<\infty$, and $(u, v)$ be a pair of weights, $\sigma=v^{1-p^{\prime}}$, and suppose that $M_{\sigma}^{\mathcal{B}}$ is bounded on $L^{p}(\sigma)$. 
Then

$$
\left\|M_{\alpha}^{\mathcal{B}} f\right\|_{L^{q}(u)} \leq C\|f\|_{L^{p}(v)}
$$

holds for all $f \in L^{p}(v)$ if and only if the pair of weights satisfies the testing condition: there exists $C>0$ such that

$$
\left(\int_{G} M_{\alpha}^{\mathcal{B}}\left(\chi_{G} \sigma\right)(x)^{q} u(x) d x\right)^{1 / q} \leq C\left(\int_{G} \sigma(x) d x\right)^{1 / p}
$$

for all $G$ that are unions of sets in $\mathcal{B}$. Furthermore, if we let

$$
[u, v]_{S_{p, q}^{\mathcal{B}}}=\sup _{G} \frac{\left(\int_{G} M_{\alpha}^{\mathcal{B}}\left(\chi_{G} \sigma\right)^{q} u d x\right)^{1 / q}}{\sigma(G)^{1 / p}}<\infty,
$$

where the supremum is over all $G$ that are unions of sets in $\mathcal{B}$, then

$$
\left\|M_{\alpha}^{\mathcal{B}}\right\|_{L^{p}(v) \rightarrow L^{q}(u)} \leq C[u, v]_{S_{p, q}^{\mathcal{B}}}\left\|M_{\sigma}^{\mathcal{B}}\right\|_{L^{p}(\sigma) \rightarrow L^{p}(\sigma)} .
$$

Proof. We prove only the case $p<q$, as the case $p=q$ is similar to Theorem B. The necessity of the testing condition follows from letting $f=\chi_{G} \sigma$. Suppose that $(u, v)$ is a pair of weights that satisfy the testing condition (4.2). Then let $\Omega_{k}=\left\{x: 2^{k}<M_{\alpha}^{\mathcal{B}} f(x) \leq 2^{k+1}\right\}$ for $k \in \mathbb{Z}$. From the definition of $M_{\alpha}^{\mathcal{B}}$, for each $k$ we get $\Omega_{k} \subseteq \bigcup_{j=1}^{\infty} B_{k, j}$ where $B_{k, j}$ satisfies

$$
\frac{1}{\left|B_{k, j}\right|^{1-\alpha / n}} \int_{B_{k, j}}|f(y)| d y>2^{k} .
$$

For each $k$ let $E_{k, 1}=B_{k, 1} \cap \Omega_{k}$ and for $j>1$ define

$$
E_{k, j}=\left(B_{k, j} \backslash \bigcup_{i=1}^{j-1} B_{k, j}\right) \cap \Omega_{k} .
$$

Notice that for each $k$ the collection $\left\{E_{k, j}\right\}_{j}$ is disjoint and since the $\Omega_{k}$ 's are disjoint, the $E_{k, j}$ 's are disjoint for all $k, j$. Further, $\Omega_{k}=\bigcup_{j} E_{k, j}$, hence we may estimate $\left\|M_{\alpha}^{\mathcal{B}} f\right\|_{L^{q}(u)}$. We have

$$
\begin{aligned}
\int_{\mathbb{R}^{n}}\left(M_{\alpha}^{\mathcal{B}} f(x)\right)^{q} u(x) d x \leq C \sum_{k, j} u\left(E_{k, j}\right)\left(\frac{1}{\left|B_{k, j}\right|^{1-\alpha / n}} \int_{B_{k, j}}|f(y)| d y\right)^{q} \\
\quad=C \sum_{k, j} u\left(E_{k, j}\right)\left(\frac{\sigma\left(B_{k, j}\right)}{\left|B_{k, j}\right|^{1-\alpha / n}}\right)^{q}\left(\frac{1}{\sigma\left(B_{k, j}\right)} \int_{B_{k, j}}|f| \sigma^{-1} \sigma d y\right)^{q}=C \int_{X} g d \mu
\end{aligned}
$$

where $X=\mathbb{Z} \times \mathbb{N}, g$ is the function on $X$ defined by

$$
g(k, j)=\left(\frac{1}{\sigma\left(B_{k, j}\right)} \int_{B_{k, j}}|f| \sigma^{-1} \sigma d y\right)^{q},
$$

and $\mu$ is a discrete measure on $X$ with 


$$
\mu(k, j)=u\left(E_{k, j}\right)\left(\frac{\sigma\left(B_{k, j}\right)}{\left|B_{k, j}\right|^{1-\alpha / n}}\right)^{q} .
$$

Let

$$
\Gamma_{\lambda}=\{(k, j) \in X: g(k, j)>\lambda\} \quad \text { and } \quad G_{\lambda}=\bigcup\left\{B_{k, j}:(k, j) \in \Gamma_{\lambda}\right\} .
$$

We estimate $\mu\left(\Gamma_{\lambda}\right)$ using the testing condition (4.2). We have

$$
\begin{aligned}
\mu\left(\Gamma_{\lambda}\right) & =\sum_{(k, j) \in \Gamma_{\lambda}} u\left(E_{k, j}\right)\left(\frac{\sigma\left(B_{k, j}\right)}{\left|B_{k, j}\right|^{1-\alpha / n}}\right)^{q} \\
& \leq \sum_{(k, j) \in \Gamma_{\lambda}} \int_{E_{k, j}} M_{\alpha}^{\mathcal{B}}\left(\chi_{B_{k, j}} \sigma\right)^{q} u d x \\
& \leq \int_{G_{\lambda}} M_{\alpha}^{\mathcal{B}}\left(\chi_{G_{\lambda}} \sigma\right)^{q} u d x \leq[u, v]_{S_{p, q}^{\mathcal{B}}}^{q} \sigma\left(G_{\lambda}\right)^{q / p} \\
& \leq C[u, v]_{S_{p, q}^{\mathcal{B}}}^{q} \sigma\left\{x: M_{\sigma}^{\mathcal{B}}(f / \sigma)(x)^{q}>\lambda\right\}^{q / p} .
\end{aligned}
$$

Now we proceed with estimating $\int_{X} g d \mu$ :

$$
\begin{aligned}
\int_{X} g d \mu & =\int_{0}^{\infty} \mu\left(\Gamma_{\lambda}\right) d \lambda \\
& \leq C[u, v]_{S_{p, q}^{\mathcal{B}}}^{q} \int_{0}^{\infty} \sigma\left\{x: M_{\sigma}^{\mathcal{B}}(f / \sigma)(x)^{q}>\lambda\right\}^{q / p} d \lambda \\
& =C[u, v]_{S_{p, q}^{\mathcal{B}}}^{q} \int_{0}^{\infty}\left(t \sigma\left\{x: M_{\sigma}^{\mathcal{B}}(f / \sigma)(x)^{p}>t\right\}\right)^{q / p} \frac{d t}{t} .
\end{aligned}
$$

Since $p<q$, we use the fact that the measure $d t / t$ on $(0, \infty)$ is essentially a counting measure. Continuing,

$$
\begin{aligned}
\int_{0}^{\infty}(t \sigma & \left.\left\{x: M_{\sigma}^{\mathcal{B}} f(x)^{p}>t\right\}\right)^{q / p} \frac{d t}{t}=\sum_{l \in \mathbb{Z}} \int_{2^{l}}^{2^{l+1}}\left(t \sigma\left\{x: M_{\sigma}^{\mathcal{B}} f(x)^{p}>t\right\}\right)^{q / p} \frac{d t}{t} \\
& \leq 2^{q / p} \log 2 \sum_{l \in \mathbb{Z}}\left(2^{l} \sigma\left\{x: M_{\sigma}^{\mathcal{B}}(f / \sigma)(x)^{p}>2^{l}\right\}\right)^{q / p} \\
& \leq C\left(\sum_{l \in \mathbb{Z}} \sigma\left\{x: M_{\sigma}^{\mathcal{B}}(f / \sigma)(x)^{p}>2^{l}\right\} 2^{l}\right)^{q / p} \\
& \leq C\left(\sum_{l \in \mathbb{Z}} \int_{2^{l-1}}^{2^{l}} \sigma\left\{x: M_{\sigma}^{\mathcal{B}}(f / \sigma)(x)^{p}>t\right\} d t\right)^{q / p} \\
& =C\left(\int_{0}^{\infty} \sigma\left\{x: M_{\sigma}^{\mathcal{B}}(f / \sigma)(x)^{p}>t\right\} d t\right)^{q / p} \leq C\left\|M_{\sigma}^{\mathcal{B}}\right\|^{q}\left(\int_{\mathbb{R}^{n}}|f|^{p} v d x\right)^{q / p} .
\end{aligned}
$$


This finishes the proof of Theorem 4.1, and if one keeps track of the constants, one can easily see that

$$
\left\|M_{\alpha}^{\mathcal{B}}\right\| \leq C[u, v]_{S_{p, q}^{\mathcal{B}}}\left\|M_{\sigma}^{\mathcal{B}}\right\|
$$

We state two corollaries of Theorem 4.1 for the bases $\mathcal{Q}$ and $\mathcal{D}$. We start with the basis $\mathcal{D}$ and employ an argument similar to the one found in $[5$, p. 430]. We have the following dyadic version of Sawyer's theorem.

Corollary 4.2. Let $0 \leq \alpha<n$ and $1<p \leq q<\infty$ and $(u, v)$ be a pair of weights with $\sigma=v^{1-p^{\prime}}$. Then

$$
\left\|M_{\alpha}^{\mathcal{D}} f\right\|_{L^{q}(u)} \leq C\|f\|_{L^{p}(v)}
$$

if and only if $(u, v)$ satisfies the testing condition

$$
[u, v]_{S_{p, q}^{d}}=\sup _{Q \in \mathcal{D}} \frac{\left(\int_{Q} M_{\alpha}^{\mathcal{D}}\left(\chi_{Q} \sigma\right)(x)^{q} u(x) d x\right)^{1 / q}}{\sigma(Q)^{1 / p}}<\infty .
$$

Further, we have the following dependence on the operator norm:

$$
\left\|M_{\alpha}^{\mathcal{D}}\right\| \leq C[u, v]_{S_{p, q}^{d}} .
$$

Proof. The necessity of the condition (4.3) is clear. Note that $M_{\sigma}^{\mathcal{D}}$ is bounded on $L^{p}(\sigma)$ with $\left\|M_{\sigma}^{\mathcal{D}}\right\| \leq C_{n, p}$. We will show that $(u, v)$ satisfies the testing condition

$$
\left(\int_{G} M_{\alpha}^{\mathcal{D}}\left(\chi_{G} \sigma\right)^{q} u d x\right)^{1 / q} \leq c[u, v]_{S_{p, q}^{d}} \sigma(G)^{1 / p}
$$

for $G$ a union of dyadic cubes, hence showing $[u, v]_{S_{p, q}^{\mathcal{D}}} \leq c[u, v]_{S_{p, q}^{d}}$.

We will actually show this inequality for the truncated version of $M_{\alpha}^{\mathcal{D}}$. Let $M_{\alpha}^{N}$ be the same operator as $M_{\alpha}^{\mathcal{D}}$ except with supremum over all dyadic cubes of side length less than or equal to $2^{N}$. We show (4.4) with $M_{\alpha}^{\mathcal{D}}$ replaced by $M_{\alpha}^{N}$ and constant independent of $N$. Let $G$ be a union of dyadic cubes. Using the same discretization as in Theorem 4.1, we may write $\left\{x: M_{\alpha}^{N}\left(\chi_{G} \sigma\right)(x)>2^{k}\right\}=\bigcup_{j} Q_{k, j}$ where $Q_{k, j}$ are maximal dyadic (hence disjoint for a fixed $k$ ) cubes of side length less than or equal to $2^{N}$ that are contained in $G$ and satisfy

$$
\frac{\sigma\left(Q_{k, j}\right)}{\left|Q_{k, j}\right|^{1-\alpha / n}}>2^{k}
$$

If we let

$$
E_{k, j}=Q_{k, j} \cap\left\{x: 2^{k}<M_{\alpha}^{N}\left(\chi_{G} \sigma\right) \leq 2^{k+1}\right\},
$$

then the $E_{k, j}$ 's are disjoint for all $k$ and $j$ with

$$
\left\{x: 2^{k}<M_{\alpha}^{N}\left(\chi_{G} \sigma\right) \leq 2^{k+1}\right\}=\bigcup_{j} E_{k, j} .
$$


Thus, continuing as in Theorem 4.1 we have

$$
\int_{G} M_{\alpha}^{N}\left(\chi_{G} \sigma\right)^{q} u d x \leq C \sum_{k, j} u\left(E_{k, j}\right)\left(\frac{\sigma\left(Q_{k, j}\right)}{\left|Q_{k, j}\right|^{1-\alpha / n}}\right)^{q} .
$$

Since the $Q_{k, j}$ 's are dyadic cubes with side length less than $2^{N}$ we can extract a maximal disjoint collection of them, say $\left\{Q_{i}\right\}$. We have

$$
\begin{aligned}
\sum_{k, j} u\left(E_{k, j}\right)\left(\frac{\sigma\left(Q_{k, j}\right)}{\left|Q_{k, j}\right|^{1-\alpha / n}}\right)^{q} & \leq \sum_{i} \sum_{Q_{k, j} \subseteq Q_{i}} u\left(E_{k, j}\right)\left(\frac{\sigma\left(Q_{k, j}\right)}{\left|Q_{k, j}\right|^{1-\alpha / n}}\right)^{q} \\
& \leq \sum_{i} \sum_{Q_{k, j} \subseteq Q_{i}} \int_{E_{k, j}} M_{\alpha}\left(\chi_{Q_{k, j}} \sigma\right)^{q} u d x \\
& \leq \sum_{i} \int_{Q_{i}} M_{\alpha}\left(\chi_{Q_{i}} \sigma\right)^{q} u d x \\
& \leq[u, v]_{S_{p, q}^{d}}^{q} \sum_{i} \sigma\left(Q_{i}\right)^{q / p} \leq[u, v]_{S_{p, q}^{d}}^{q} \sigma(G)^{q / p}
\end{aligned}
$$

Finally, we may obtain the full version of Sawyer's theorem using Lemma 2.2. We have the following corollary.

Corollary 4.3. Suppose that $0 \leq \alpha<n, 1<p \leq q<\infty$, and $(u, v)$ is a pair of weights with $\sigma=v^{1-p^{\prime}}$. Then

$$
\left\|M_{\alpha} f\right\|_{L^{q}(u)} \leq C\|f\|_{L^{p}(v)}
$$

for all $f$ if and only if $(u, v)$ satisfies

$$
[u, v]_{S_{p, q}}=\sup _{Q} \frac{\left(\int_{Q} M_{\alpha}\left(\chi_{Q} \sigma\right)^{q} u d x\right)^{1 / q}}{\sigma(Q)^{1 / p}}<\infty,
$$

and

$$
\left\|M_{\alpha}\right\| \leq C[u, v]_{S_{p, q}} .
$$

Proof. First notice that if $(u, v)$ satisfies condition (4.5), then $\left(\tau_{t} u, \tau_{t} v\right)$ satisfies the dyadic condition $S_{p, q}^{d}$ with

$$
\sup _{t}\left[\tau_{t} u, \tau_{t} v\right]_{S_{p, q}^{d}} \leq[u, v]_{S_{p, q}} .
$$

Combining Corollary 4.2 and Lemma 2.2 we have

$$
\begin{aligned}
\left\|M_{\alpha} f\right\|_{L^{q}(u)} & \leq C \sup _{t}\left\|\tau_{-t} \circ M_{\alpha} \circ \tau_{t} f\right\|_{L^{q}(u)} \leq C \sup _{t}\left[\tau_{t} u, \tau_{t} v\right]_{S_{p, q}^{d}}\left\|\tau_{t} f\right\|_{L^{p}\left(\tau_{t} v\right)} \\
& \leq C[u, v]_{S_{p, q}}\|f\|_{L^{p}(v)} \text {. }
\end{aligned}
$$

We now give a testing condition for $M_{\alpha}^{\mathcal{B}}$ that is more natural when $\alpha>0$ and also yields sharp operator norms in the one-weight case. We have the following result. 
Theorem 4.4. Suppose that $0 \leq \alpha<n, 1<p, q<\infty$, and $(u, v)$ is a pair of weights such that $M_{\alpha, \sigma}^{\mathcal{B}}$ is bounded from $L^{p}(\sigma)$ to $L^{q}(\sigma)$, and that satisfy the testing condition

$$
\left(\int_{Q} M^{\mathcal{B}}\left(\chi_{G} \sigma\right)^{(1-\alpha / n) q} u d x\right)^{1 / q} \leq C \sigma(G)^{1 / q}
$$

for all $G$ that are unions of sets in $\mathcal{B}$. If $[u, v]_{T_{q}^{\mathcal{B}}}$ denotes the smallest constant that satisfies (4.6) for all such $G$, then

$$
\left\|M_{\alpha}^{\mathcal{B}}\right\|_{L^{q}(u) \rightarrow L^{p}(v)} \leq C[u, v]_{T_{q}^{\mathcal{B}}}\left\|M_{\alpha, \sigma}^{\mathcal{B}}\right\|_{L^{p}(\sigma) \rightarrow L^{q}(\sigma)} .
$$

Before we present the proof, some remarks are in order. First notice that condition (4.6) is just a sufficient condition for the boundedness of $M_{\alpha}^{\mathcal{B}}$. It is not known if this is also necessary since the testing condition is based on testing $M^{\mathcal{B}}$ and not $M_{\alpha}^{\mathcal{B}}$. When $\alpha=0$ and $p=q$ the two conditions (4.2) and (4.6) are the same and thus we once again recover Jawerth's result, Theorem B. Further, notice that we do not have the restriction $p \leq q$ but we do need $M_{\alpha, \sigma}^{\mathcal{B}}$ to be bounded from $L^{p}(\sigma)$ to $L^{q}(\sigma)$, which usually happens when $1 / p-1 / q=\alpha / n$.

Proof of Theorem 4.4. We use the same basic discretization of Jawerth as in Theorem 4.1 to obtain

$$
\begin{aligned}
\int_{\mathbb{R}^{n}} & M_{\alpha}^{\mathcal{B}} f^{q} u d x \leq C \sum_{k, j} u\left(E_{k, j}\right)\left(\frac{1}{\left|B_{k j}\right|^{1-\alpha / n}} \int_{B_{k, j}}|f| d x\right)^{q} \\
& =C \sum_{k, j} u\left(E_{k, j}\right)\left(\frac{\sigma\left(B_{k, j}\right)}{\left|B_{k, j}\right|}\right)^{(1-\alpha / n) q}\left(\frac{1}{\sigma\left(B_{k, j}\right)^{1-\alpha / n}} \int_{B_{k, j}}|f| \sigma^{-1} \sigma\right)^{q} \\
& =C \int_{X} g d \mu .
\end{aligned}
$$

Here $X, g$ and $\mu$ are defined analogously to those in the proof Theorem 4.1. The definitions for $\Gamma_{\lambda}$ and $G_{\lambda}$ are also exactly as in that proof. Then

$$
\begin{aligned}
\mu\left(\Gamma_{\lambda}\right) & =\sum_{(k, j) \in \Gamma_{\lambda}} u\left(E_{k, j}\right)\left(\frac{\sigma\left(B_{k, j}\right)}{\left|B_{k, j}\right|}\right)^{(1-\alpha / n) q} \leq \int_{G_{\lambda}} M^{\mathcal{B}}\left(\chi_{G_{\lambda}} \sigma\right)^{(1-\alpha / n) q} u d x \\
& \leq[u, v]_{T_{q}^{\mathcal{B}}}^{q} \sigma\left(G_{\lambda}\right) \leq[u, v]_{T_{q}^{\mathcal{B}}}^{q} \sigma\left(\left\{x: M_{\alpha, \sigma}^{\mathcal{B}}(f / \sigma)(x)^{q}>\lambda\right\}\right) .
\end{aligned}
$$

Plugging this into the estimate for $M_{\alpha}^{\mathcal{B}}$ we have

$$
\begin{aligned}
\int_{X} g d \mu & =\int_{0}^{\infty} \mu\left(\Gamma_{\lambda}\right) d \lambda \leq[u, v]_{T_{q}^{\mathcal{B}}}^{q} \int_{0}^{\infty} \sigma\left(\left\{x: M_{\alpha, \sigma}^{\mathcal{B}}(f / \sigma)(x)^{q}>\lambda\right\}\right) d \lambda \\
& =[u, v]_{T_{q}^{\mathcal{B}}}^{q} \int_{\mathbb{R}^{n}} M_{\alpha, \sigma}^{\mathcal{B}}(f / \sigma)^{q} \sigma d x \leq[u, v]_{T_{q}^{\mathcal{B}}}^{q}\left\|M_{\alpha, \sigma}^{\mathcal{B}}\right\|^{q}\left(\int_{\mathbb{R}^{n}}|f|^{p} v d x\right)^{q / p} \cdot \mathbf{-}
\end{aligned}
$$


We also note that if $p$ and $q$ are related by the equation $1 / q=1 / p-\alpha / n$ and $\mathcal{B}=\mathcal{D}$ then the boundedness of $M_{\alpha, \sigma}^{\mathcal{D}}$ follows from Lemma 2.1. Once again we may relax the testing conditions in the case $\mathcal{B}=\mathcal{D}$ or $\mathcal{Q}$. We obtain the following corollaries which are similar to Corollaries 4.2 and 4.3 , and we state them without proof.

Corollary 4.5. Suppose $1<p<n / \alpha$ and $1 / q=1 / p-\alpha / n$. If $(u, v)$ is a pair of weights that satisfies

$$
[u, v]_{T_{q}^{d}}=\sup _{Q \in \mathcal{D}} \frac{\left(\int_{Q} M^{\mathcal{D}}\left(\chi_{Q} \sigma\right)^{1+q / p^{\prime}} u d x\right)^{1 / q}}{\sigma(Q)^{1 / q}}<\infty,
$$

then $M_{\alpha}^{\mathcal{D}}$ maps $L^{p}(v)$ into $L^{q}(u)$ with

$$
\left\|M_{\alpha}^{\mathcal{D}}\right\| \leq C[u, v]_{T_{q}^{d}} .
$$

Using Lemma 2.2 we may pass this result to the basis of cubes.

Corollary 4.6. Suppose $1<p<n / \alpha$ and $1 / q=1 / p-\alpha / n$. If $(u, v)$ is a pair of weights that satisfies

$$
[u, v]_{T_{q}}=\sup _{Q} \frac{\left(\int_{Q} M\left(\chi_{Q} \sigma\right)^{1+q / p^{\prime}} u d x\right)^{1 / q}}{\sigma(Q)^{1 / q}}<\infty,
$$

then $M_{\alpha}$ maps $L^{p}(v)$ into $L^{q}(u)$ with

$$
\left\|M_{\alpha}\right\| \leq C[u, v]_{T_{q}} .
$$

When $\alpha>0,(4.7)$ is a new sufficient condition for the two-weight boundedness of $M_{\alpha}$. Instead of testing $M_{\alpha}$, one needs to test $M$ to obtain the two-weight boundedness of $M_{\alpha}$. Clearly it is stronger than the testing condition (4.5), but it does give the sharp constant for the one-weight case (see below).

5. Sharp bounds. We remarked in the introduction that when $\mathcal{B}=\mathcal{Q}$ the sharp dependence on the operator norm of $M_{\alpha}$ is given by

$$
\left\|M_{\alpha}\right\| \leq C[w]_{A_{p, q}}^{\frac{p^{\prime}}{q}(1-\alpha / n)} .
$$

This is shown in [8] using techniques similar to those in [9]. The sharpness is also shown in [8] by using families of power functions and power weights. It should also be noted that (5.1) follows from combining Lemma 2.2 and Corollary 3.4. We give a different proof of (5.1) using the two-weight dependence of Corollary 4.6. First, we examine the relationship between the two-weight $T_{q}$ constant and the one-weight $A_{p, q}$ constant. We use a similar approach to that of Hunt, Kurtz, and Neugebauer [6]. 
Theorem 5.1. Let $0 \leq \alpha<n, 1<p<n / \alpha, 1 / q=1 / p-\alpha / n$, and $w$ be a weight for the basis $\mathcal{Q}$. Then

$$
[w]_{A_{p, q}} \leq\left[w^{q}, w^{p}\right]_{T_{q}}^{q} \leq C[w]_{A_{p, q}}^{p^{\prime}(1-\alpha / n)} .
$$

Proof. Let $w$ be a weight, $u=w^{q}$, and $v=w^{p}$, so that $\sigma=w^{-p^{\prime}}$. First notice that

$$
u(Q)\left(\frac{\sigma(Q)}{|Q|}\right)^{1+q / p^{\prime}} \leq \int_{Q} M\left(\chi_{Q} \sigma\right)^{1+q / p^{\prime}} u d x \leq[u, v]_{T_{q}}^{q} \sigma(Q) .
$$

This shows that $[w]_{A_{p, q}} \leq\left[w^{q}, w^{p}\right]_{T_{q}}^{q}$. On the other hand, let $Q$ be a cube and notice that

$$
M\left(\chi_{Q} \sigma\right)(x)=\sup _{P \ni x} \frac{1}{|P|} \int_{P} \sigma d x
$$

where the supremum is over all cubes $P$ containing $x$ and contained in $Q$. Suppose $w \in A_{p, q}, x \in Q$ and $P \subseteq Q$ is a cube containing $x$. Then

$$
\begin{aligned}
\left(\frac{\sigma(P)}{|P|}\right)^{1+q / p^{\prime}} & \leq C_{n}[w]_{A_{p, q}}^{p^{\prime}(1-\alpha / n)}\left(\frac{1}{u(3 P)} \int_{P} u^{-1} \chi_{Q} u\right)^{1+p^{\prime} / q} \\
& \leq C[w]_{A_{p, q}}^{p^{\prime}(1-\alpha / n)} M_{u}^{c}\left(\chi_{Q} u^{-1}\right)(x)^{1+p^{\prime} / q} .
\end{aligned}
$$

It follows that $M\left(\chi_{Q} \sigma\right)(x)^{1+q / p^{\prime}} \leq C[w]_{A_{p, q}}^{p^{\prime}(1-\alpha / n)} M_{u}^{c}\left(\chi_{Q} u^{-1}\right)(x)^{1+p^{\prime} / q}$ for all $x \in Q$. Plugging this into the testing condition and using the fact that $M_{u}^{c}$ is bounded on $L^{1+p^{\prime} / q}(u)$ with norm independent of $u$, we have

$$
\begin{aligned}
\int_{Q} M\left(\chi_{Q} \sigma\right)^{1+q / p^{\prime}} u d x & \leq C[w]_{A_{p, q}}^{p^{\prime}(1-\alpha / n)} \int_{\mathbb{R}^{n}} M_{u}^{c}\left(\chi_{Q} u^{-1}\right)^{1+p^{\prime} / q} u d x \\
& \leq C_{n}[w]_{A_{p, q}}^{p^{\prime}(1-\alpha / n)} \int_{Q} u^{-1-p^{\prime} / q} u d x \\
& =C_{n}[w]_{A_{p, q}}^{p^{\prime}(1-\alpha / n)} \sigma(Q) .
\end{aligned}
$$

Hence we obtain the sharp bound (1.2) found in [8]. We have

$$
\left\|M_{\alpha}\right\|_{L^{p}\left(w^{p}\right) \rightarrow L^{q}\left(w^{q}\right)} \leq C\left[w^{q}, w^{p}\right]_{T_{p}} \leq[w]_{A_{p, q}}^{p^{\prime} / q(1-\alpha / n)} .
$$

To conclude we make some remarks about the consequences of Corollary 4.6 and Theorem 5.1 when $\alpha=0$. In this case we have $p=q$ and the testing conditions $T_{p}$ and $S_{p}$ are the same. If we renormalize back to $w$ $\left(w^{p} \mapsto w\right)$ and write $[w]_{S_{p}}$ for $[w, w]_{S_{p}}$, then inequality (5.2) in Theorem 5.1 becomes

$$
[w]_{A_{p}}^{1 / p} \leq[w]_{S_{p}} \leq C[w]_{A_{p}}^{1 /(p-1)} .
$$

The second inequality has a few interesting consequences. First, it leads to a new proof of Buckley's estimate (1.1). Indeed, using (4.8) in Corol- 
lary 4.6 we obtain

$$
\|M\|_{L^{p}(w) \rightarrow L^{p}(w)} \leq C[w]_{S_{p}} \leq C[w]_{A_{p}}^{1 /(p-1)} .
$$

As noted in the introduction, this is basically combining Sawyer's two-weight result with a variation of the arguments of Hunt, Kurtz, and Nuegebauer. Second, the operator norm dependence for the two-weight case is sharp, i.e. the inequality

$$
\|M\|_{L^{p}(v) \rightarrow L^{p}(u)} \leq C[u, v]_{S_{p}}
$$

is sharp. This follows from the one-weight case, since if we had a better bound in (5.4), then taking $u=v=w \in A_{p}$ and using (5.3) would imply a better bound in (1.1). Finally, the second inequality in (5.3) is sharp. Once again, a better bound in the second inequality in (5.3) would imply a sharper bound in (1.1).

Acknowledgments. The author would like to thank Rodolfo H. Torres and Carlos Pérez for their many suggestions and discussions that improved the quality of this paper. The author also benefitted from a very detailed write-up on the history of weighted inequalities for maximal operators given by a referee of an earlier draft of this paper.

Research supported in part by the National Science Foundation under grants DMS 0400423 and DMS 0800492.

\section{References}

[1] S. Buckley, Estimates for operator norms on weighted spaces and reverse Jensen inequalities, Trans. Amer. Math. Soc. 340 (1993), 253-272.

[2] M. Christ and R. Fefferman, A note on weighted norm inequalities for the HardyLittlewood maximal operator, Proc. Amer. Math. Soc. 87 (1983), 447-448.

[3] R. Coifman and C. Fefferman, Weighted norm inequalities for maximal functions and singular integrals, Studia Math. 51 (1974), 241-250.

[4] C. Fefferman and E. Stein, Some maximal inequalities, Amer. J. Math. 93 (1971), 107-115.

[5] J. García-Cuerva and J. L. Rubio de Francia, Weighted Norm Inequalities and Related Topics, North-Holland Math. Stud. 116, North-Holland, Amsterdam, 1985.

[6] R. Hunt, D. Kurtz and C. Neugebauer, A note on the equivalence of $A_{p}$ and Sawyer's condition for equal weights, in: Proc. Conf. on Harmonic Analysis in Honor of A. Zygmund, Wadsworth Math. Ser., Wadsworth, Belmont, CA, 1983, Vol. 1, 156-158.

[7] B. Jawerth, Weighted inequalities for maximal operators: linearization, localization and factorization, Amer. J. Math. 108 (1986), 361-414.

[8] M. Lacey, K. Moen, C. Pérez and R. H. Torres, Sharp weighted bounds for fractional integral operators, submitted, 2009.

[9] A. Lerner, An elementary approach to several results on the Hardy-Littlewood maximal operator, Proc. Amer. Math. Soc. 136 (2008), 2829-2833.

[10] B. Muckenhoupt, Weighted norm inequalities for the Hardy maximal function, Trans. Amer. Math. Soc. 165 (1972), 207-226. 
[11] B. Muckenhoupt and R. Wheeden, Weighted norm inequalities for fractional integrals, Trans. Amer. Math. Soc. 192 (1974), 261-274.

[12] E. T. Sawyer, A characterization of a two-weight norm inequality for maximal operators, Studia Math. 75 (1982), 1-11.

Department of Mathematics

University of Kansas

405 Snow Hall

1460 Jayhawk Blvd.

Lawrence, KS 66045-7523, U.S.A.

E-mail: moen@math.ku.edu 